\title{
A STUDY ON IMPACT OF ALCOHOL AMONG YOUNG INDIAN POPULATION USING HRV ANALYSIS
}

\author{
D Mahesh Kumar ${ }^{1}$, Dr.Prasanna Kumar S.C ${ }^{2}$, Dr.B.G Sudarshan ${ }^{3}$, Yadhuraj S.R ${ }^{4}$ \\ ${ }^{1}$ Assistant Professor, Dept of Electronics and Instrumentation Engineering JSSATE, PhD \\ Scholar at Jain University Bangalore, India \\ ${ }^{2}$ Professor \& HOD, Dept of Electronics and Instrumentation Engineering ,RVCE \\ ,Bangalore \\ ${ }^{3}$ Assosiate Professor, Dept of Electronics and Instrumentation Engineering, RVCE \\ ,Bangalore \\ ${ }^{4}$ Ph.D Scholar, Dept of Electronics and Instrumentation Engineering, RVCE ,Bangalore
}

\begin{abstract}
Heart Rate Variability (HRV) is the measure of time difference between two successive heart beats and its variation occurring due to internal and external stimulation causes. HRV is a non-invasive tool for indirect investigation of both cardiac and autonomic system function in both healthy and diseased condition. It has been speculated that HRV analysis by nonlinear method might bring potentially useful prognosis information into light which will be helpful for assessment of cardiac condition. In this study, HRV from two types of data sets are analyzed which are collected from different subjects in the age group of 18 to 22. Then parameters of linear methods and three nonlinear methods, approximate entropy (ApEn), detrended fluctuation analysis (DFA) and Poincare plot have been applied to analyze HRV among 158 subjects of which 79 are control study and 79 are alcoholics. It has been clearly shown that the linear and nonlinear parameters obtained from these two methods reflect the opposite heart condition of the two types of data under study among alcoholics non-alcoholic's by HRV measures. Poincare plot clearly distinguishes between the alcoholics by analysing the location of points in the ellipse of the Poincare plot. In alcoholics the points of the Poincare plot will be concentrated at the centre of the ellipse and in nonalchoholics the points will be much concentrated along the periphery of the ellipse. The Approximate Entropy value will be lesser than one in alcoholics and in nonalcoholics the entropy shows values greater than one. The increased LF/HF value in alcoholics denotes the increase in sympathetic nervous system activities and decrease of the parasympathetic activity which will be lesser in alcoholics subjects.
\end{abstract}

\section{KEYWORDS}

ECG, HRV, Alcoholic, Approximate Entropy, ANS, Poincare Plot

\section{INTRODUCTION}

Changes in environmental conditions, emotional reactions, thoughts - all these and external and internal stimulations immediately change heart rate. Heart beat comes slightly early, or late. This phenomenon is termed as "Heart Rate Variability" (HRV). Therefore, HRV is a physiological condition where the time interval between heart beats varies. All the organ systems of the body are controlled by Autonomic Nervous System (ANS). It also helps in maintaining homeostasis. ANS consists of two subsystems: Sympathetic and Parasympathetic Nervous System. SNS helps to prepare the body for action. When a person is under challenging situations, SNS produces so called "flight or fight" response. PNS, on the other hand, is more active under unchallenging

DOI : 10.5121/ijcseit.2014.4406 
situations, such as rest and digestion. It tends to work in opposite direction of SNS, bringing the body towards a rest state. SNS activation increases heart rate and breathing rate, but PNS decreases it. Constant interaction of these two systems is reflected in HRV. Therefore, HRV can be used to estimate the activity and activation level of both systems. Indirect investigation of both cardiac and autonomic system function in both healthy and diseased condition is possible by means of HRV analysis. HRV provides various features for distinguishing heart rate under healthy and life threatening condition. There are three important approaches in measuring the parameters for HRV analysis -

1) Time domain analysis for accessing the parameter of standard deviation of normal to normal intervals (SDNN)

2) Frequency domain analysis for accessing the parameter of Power Spectral Density (PSD)

3) Nonlinear methods for accessing parameters in nonlinear considering the non stationary nature of the ECG signal

A physiological signal doesn't vary in a regular manner which introduces complexity to the signals. Linear statistical parameters and spectra of such signals will only provide the linear parameters which assume the signals are periodic. But the physiological signals generally are quasi-periodic and are nonlinear. To address the nonlinear parameters nonlinear methods are appropriate [1]. It has been conjectured that HRV analysis by nonlinear method might shed light on unexplored horizons for assessment of sudden death. The nonlinearity of the HRV are measured using the following parameters in various studies. A) 1/f slope [2], B)approximate entropy (ApEn) [3] and C) detrended fluctuation analysis [4].In this study, linear and three nonlinear methods, approximate entropy (ApEn), detrended fluctuation analysis (DFA) and Poincare plot have been applied to analyze HRV of alcoholic and non-alcoholic subjects. It is evidently shown that the contrary heart condition of the two types of subjects under study, healthy and diseased, is reflected by nonlinear parameter value. The results obtained from this study can thus help in detecting effects of alcoholism in a subject. Time-domain parameters used in heart rate variability analysis are using the intervals between consecutive normal $\mathrm{R}$ waves (noted as $\mathrm{N}$ ) of an electrical recording and are listed below:

1. $\mathrm{SDNN}=$ Standard deviation of all NN intervals

2. $\mathrm{RMSSD}=$ Root mean sum of the squares of differences between successive NN intervals

3 . NN50 count $=$ Number of pairs of adjacent NN intervals differing by more than $50 \mathrm{~ms}$.

4. PNN50= Percentile of all NN intervals which are greater than 50ms [5].

Frequency domain parameters are obtained by applying the discrete Fourier transformation to the NN interval time series. This provides a good estimation of the amount of variation at some specific frequencies. Several frequency bands are of interest in pathology:

High Frequency band (HF) is between 0.15 and $0.4 \mathrm{~Hz}$. HF is altered by respiration and obtained primarily from the parasympathetic nervous system.

Low Frequency band (LF) is between 0.04 and $0.15 \mathrm{~Hz}$. LF are obtained from both sympathetic and parasympathetic nervous system and it denotes the delay in the baroreceptor loop. Very Low Frequency band (VLF) lies between 0.0033 and $0.04 \mathrm{~Hz}$. The origin of VLF is still unknown, it is believed that it is caused due to thermal regulation of the human body.

Ultra Low Frequency (ULF) band is between 0 and $0.0033 \mathrm{~Hz}$. The ULF is measured only in 24hour recordings, because of its variations in day time and night time. Interesting from a medical point of view is also the LF/HF ratio that may reflect the autonomic balance $[5,6,7]$. 
In order to reduce the mistakes of wrong designing or improperly used techniques the following guidelines have been listed by Guidelines of Heart rate variability, Standards of measurement, physiological interpretation, and clinical use Task Force of The European Society of Cardiology and The North American Society of Pacing and Electrophysiology[5]. The signal/noise ratio, bandwidth, common mode rejection, etc of the ECG equipment used should satisfy the current industrial standards. The recorders should be such that the reconstructed signal should be free from amplitude and phase distortion. Phase-locked time tracking is mandatory for long-term ECG recorders using analogue magnetic media. Commercial HRV equipment used should match the technical requirements of the Section on Standard Measurement of HRV and its performance should be checked independently. To standardize the method of acquiring the data two types of data acquisition is generally recommended and should apply as required. (a) short-term recordings which is of $5 \mathrm{~min}$, acquired under stable physiological conditions. In general the signal acquired in this method is processed using frequency-domain methods, and/or (b) nominal 24-h recordings which is taken for long period of time and generally is processed by time-domain methods. In clinical studies while acquiring long-term ECGs, care should be taken that the individual subjects should be recorded under fairly similar conditions and environment. Before using time- domain or frequency-domain methods the acquired signal should be edited by visually analysing the signal and should correct the individual RR intervals and also QRS complex classifications should be verified[5,6].

\section{Detrended Fluctuation Analysis}

Detrended Fluctuation Analysis (DFA) was introduced mainly to deal with nonstationaries. DFA works better compared to other heuristic techniques, including R/S Analysis, and is successful inspite of the large window sizes because of theoretically justified estimators like the local Whittlemethod. To obtain DFA alpha 1(the short time correlation exponent) and alpha2 (the long time correlation exponent, the RR time series is first integrated and the integrated series $y(k)$ divided into segments of equal length $\mathrm{n}$. By fitting a least squares line to the RR intervals data, the trend in RR intervals in each of the segments is measured. The $x(k)$ is detrended by subtracting the local trend, $x_{n}(k)$ of the data in each segment. The average fluctuation of segment size $\mathrm{n}$ is indicated as $\mathrm{F}(\mathrm{n})$ in the equation:

$$
\mathrm{F}(\mathrm{n})=\sqrt{\frac{1}{2} \sum_{1}^{L}(x(k)-x n(k))^{2}}
$$

Where $\mathrm{L}$ denotes the total length of the heart rate signal and $\mathrm{k}$ is the heart rate signal number.

\section{Approximate entropy}

Approximate entropy (ApEn) gives the measures of complexity or irregularity in the signal [13, 40]. Higher values of ApEn indicate larger irregularity and lower values of ApEn indicates more regular signal. The ApEn is computed as follows.

First, a vectors $v_{j}$ of length $m$ is formed

$$
\mathrm{v}_{\mathrm{j}}=\left(\mathrm{RR}_{\mathrm{j}}, \mathrm{RR}_{\mathrm{j}+1}, \ldots \ldots \ldots \ldots \ldots \ldots . . . \mathrm{RR}_{\mathrm{j}+\mathrm{m}-1}\right)
$$

Where $\mathrm{L}$ is the number of measured $\mathrm{RR}$ intervals and $\mathrm{m}$ denotes the embedding dimension. The distance between these vectors can be written as the maximum absolute difference between the corresponding elements, i.e., 
International Journal of Computer Science, Engineering and Information Technology (IJCSEIT), Vol. 4, No.4, August 2014

$$
\mathrm{d}\left(\mathrm{v}_{\mathrm{j}}, \mathrm{v}_{\mathrm{k}}\right)=\max \left\{\mid \mathrm{RR}_{\mathrm{j}+\mathrm{n}}-\mathrm{RR}_{\mathrm{k}+\mathrm{n}} \| \mathrm{n}=0, \ldots, \mathrm{m}-1\right\}
$$

Next, for each $\mathrm{v}_{\mathrm{j}}$ the relative number of vectors $\mathrm{v}_{\mathrm{k}}$ for which $\mathrm{d}\left(\mathrm{v}_{\mathrm{j}} ; \mathrm{v}_{\mathrm{k}}\right) \leq \mathrm{r}$ is calculated. This index is denoted with $\mathrm{C}^{\mathrm{m}}{ }_{\mathrm{j}}(\mathrm{r})$ and can be written in the form

$$
\mathrm{C}_{\mathrm{j}}^{\mathrm{m}}(\mathrm{r})=\frac{\mathrm{ubrof}[\mathrm{wk} / \mathrm{d}(\mathrm{vink}) \mathrm{s}]}{2 \mathrm{~m} \| 1}
$$

After the normalization, the value of $\mathrm{C}^{\mathrm{m}}{ }_{\mathrm{j}}(\mathrm{r})$ is always smaller or equal to 1 . Since $\mathrm{u}_{\mathrm{j}}$ is also included in the count observe that the value will be atleast $\frac{1}{L-m+:}$. Applying natural logarithm on each $\mathrm{C}_{\mathrm{j}}^{\mathrm{m}}(\mathrm{r})$ and average over $\mathrm{j}$ to yield

$$
\Phi^{\mathrm{m}}(\mathrm{r})=\frac{1}{\Sigma-m+1} \sum_{i=:}^{L-m+1} \ln \mathrm{C}_{\mathrm{j}}^{\mathrm{m}}(\mathrm{r})
$$

Finally, we get approximate entropy as

$$
\operatorname{ApEn}(\mathrm{m}, \mathrm{r}, \mathrm{L})=\Phi^{\mathrm{m}}(\mathrm{r})-\Phi^{\mathrm{m}+1}(\mathrm{r})
$$

Thus, the estimate ApEn value depends on the length $m$ of the vectors $v_{j}$, the tolerance value $r$, and the length L. In software which are designed for HRV analysis will be having different default values of $\mathrm{m}$. In Kubios software the value of $\mathrm{m}$ is 2 . The length $\mathrm{N}$ of the data also affects ApEn. When $\mathrm{N}$ is increased the ApEn approaches its asymptotic of the data (SDNN).

\section{Poincare plot}

One of the commonly used nonlinear method to obtain the nonlinear parameters of the data is Poincare plot which is simple to interpret. The successive RR intervals correlation can be graphically represented by Poincare plot i.e. plot of $R R j+1$ as a function of $R R_{j}$. The shape of the plot denotes the features of the data. There are many approaches to fit the plot, Kubios software designed to fit an ellipse to plot in a parameterized shape. The ellipse is oriented according to the line-of-identity $\left(R_{\mathrm{j}}=\mathrm{RR}_{\mathrm{j}+1}\right)$ [12]. The short-term variability is $\mathrm{SD} 1$, standard deviation of the points which are perpendicular to the line-of-identity. By obtaining SDSD time-domain measure SD1 can be written as [12]

$$
\mathrm{SD}^{2}=\frac{1}{2} \mathrm{SDSD}^{2}
$$

SD2 is the long-term variability which is the standard deviation with the line-of-identity. By obtaining SDSD, SDNN time-domain measure SD2 can be written as

$$
\mathrm{SD}^{2}=2 \mathrm{SDNN}^{2}-\frac{1}{2} \mathrm{SDSD}^{2}
$$

Generally the Poincare plot of the first order is preferred because of its simplicity. As the order increases the dimensions of the plot also increases.

\section{MATERIAL AND METHODS}

We studied a group of 158 subjects of age grouped between 19-23 years. The main objective: to evaluate the prevalence of the autonomic cardiovascular complications in alcoholic personals. Description of the groups: questionnaires were administered for general patient information (age, 
sex, duration of disease, family history, and personal history), risk factors (smoking, sedentary behaviour), treatment, and patient's symptoms. The heart rate variability analysis was performed with the help of the three lead ECG electrodes The signals from the electrodes are amplified using a bio amplifier which comes along with Skript Electronics modules. The output of the bio amplifier is connected to the multi channel 6009 DAQ card, to acquire raw ECG signals from the output terminal of ECG recorders. The sampling rate is typically set to $256 \mathrm{~Hz}$. The acquired ECG signals were stored in NI TDMS file type for further offline analysis. DAQ is connected to the computer using an USB port for further processing of the signal. DAQ has to be configured inside Lab VIEW environment. The obtained TDMS file is converted to the txt file and the obtained ECG signal is further filtered using MATLAB. The baseline trends and power interferences noises are removed in MATLAB environment. The filtered ECG signal is fed to Kubios software to obtain the linear and nonlinear parameters of HRV.

\section{RESULTS}

ECG is one of the pioneer measures of electrical activity of the heart over a period. The signal is measured by electrodes attached to the skin. The acquired ECG signal will be corrupted by various noises like base line drifts, power line interferes and motion artifacts, the acquired signal must be filtered to remove the unwanted frequencies.

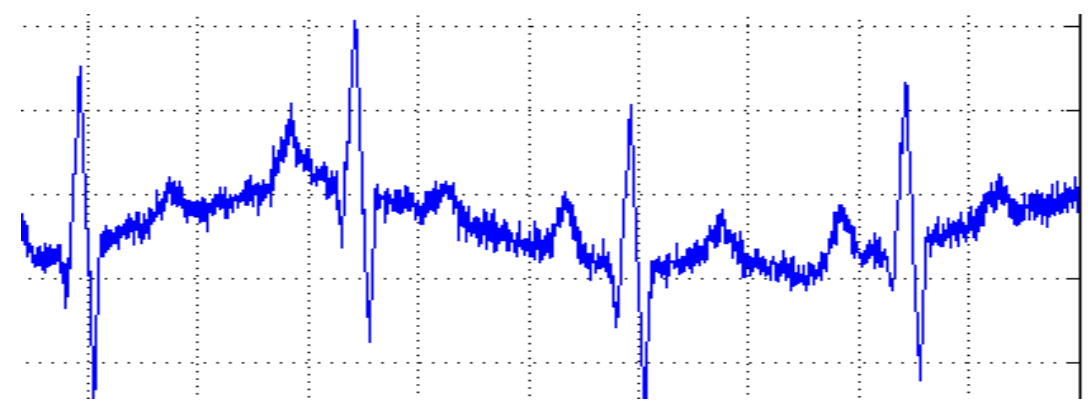

Figure1. ECG signals corrupted with trend

The above signal shows a baseline shift, we cannot consider the amplitude as actual amplitude hence, the trend in the signals should be removed first before obtaining the beat-beat intervals. In order to remove the trend, a low order polynomial is fitted to the signal and uses the polynomial to detrend it.

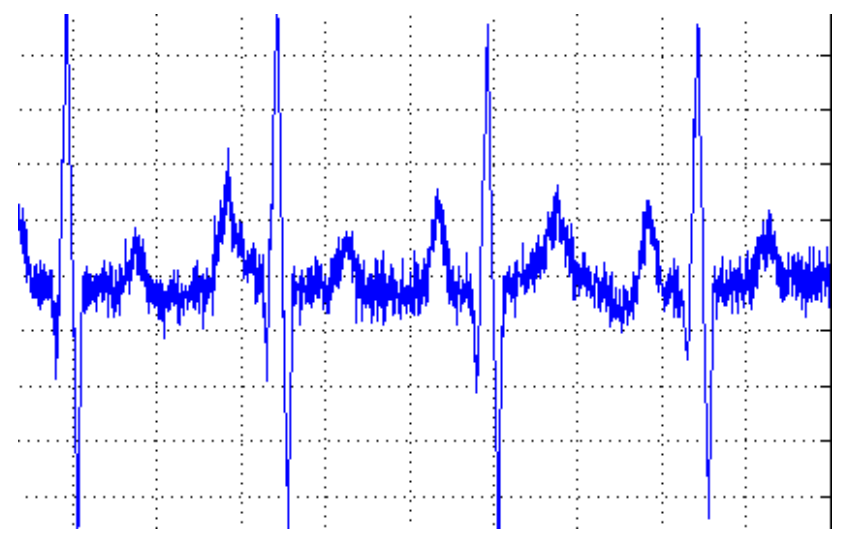

Figure2: Detrended ECG Signal 
After detrending, find the QRS-complex in the ECG signal, which is the important portion of the signal. The QRS-complex corresponds to the depolarization of the right and left ventricles of the human heart. Abnormalities in heart function and heart rate can be predicted by using QRScomplex. The peaks of the ECG signals are detected using MATLAB, which are further utilized for analyzing HRV parameters using Kubios software.

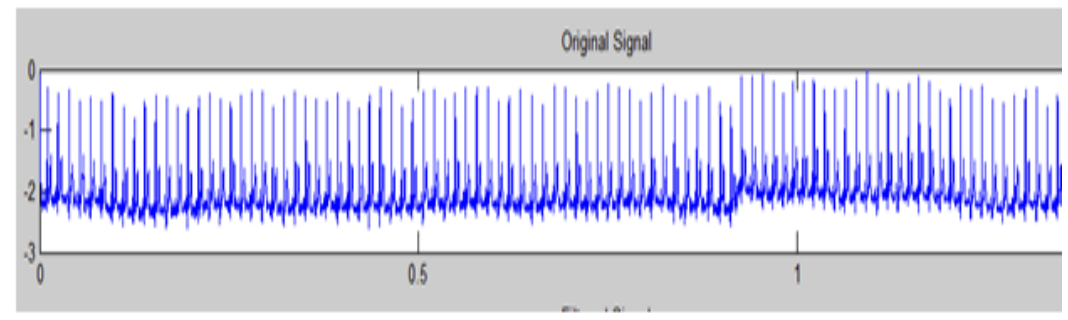

Figure 3: ECG Signal

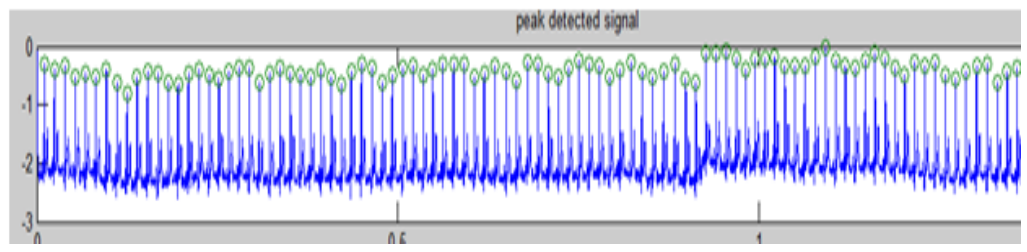

Figure 4: Peak Detected ECG Signal

\section{HRV analysis using Kubios Tool}

Kubios is an advanced software tool for studying the HRV. In this work Kubios tool is used to study the variability of the RR intervals. The time interval plot between the each RR intervals, which was obtained using MATLAB, is loaded to the Kubios tool.

\section{Time-domain analysis PRV}

The time-domain methods uses statistical analysis and are the simplest to perform since they are applied directly to the series of successive RR interval values. The results of time-domain are displayed by selecting the time-domain button on the top of the results view segment. Figure 5 shows the results of time-domain analysis of the HRV signal.

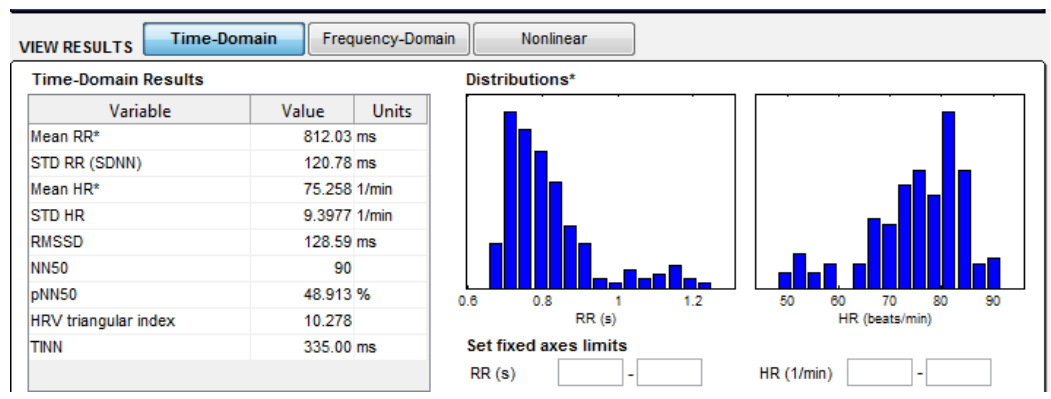

Figure 5: Results of time-domain analysis of ECG signal. 


\section{Frequency-domain Analysis}

In the frequency-domain methods, Discrete Fourier Transforms are applied to the RR interval values in time domain to obtain a power spectrum density (PSD) estimate. The results of frequency-domain are displayed by selecting the frequency-domain button on the top of the results view segment. The frequency-domain results of HRV are shown in Figure 6.

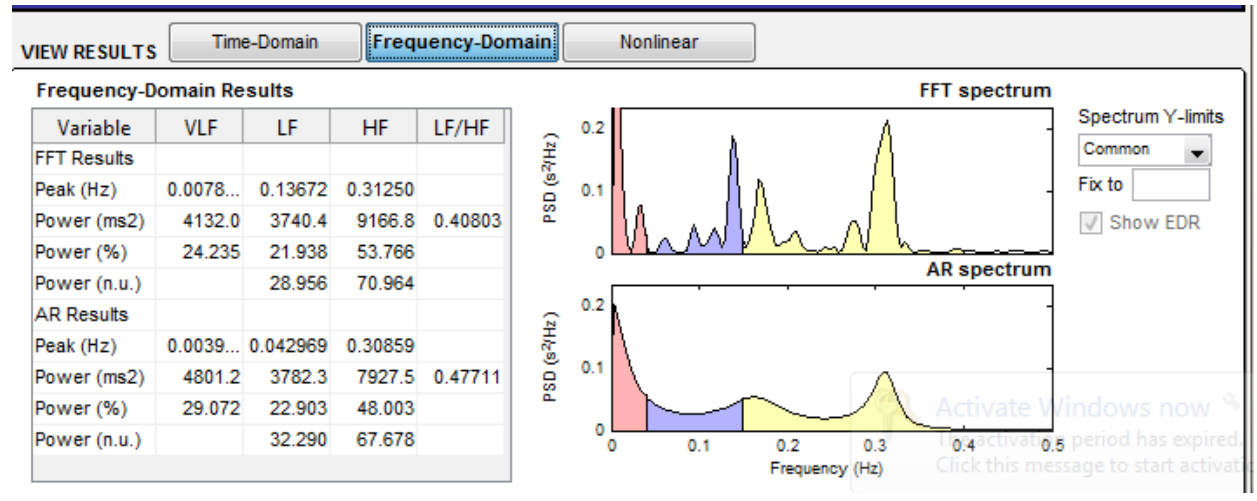

Figure 6: Results of frequency-domain analysis of ECG signal.

\section{Nonlinear Analysis}

The nonlinear analysis has been analyzed using measures such as Poincare plot, detrended fluctuation analysis, approximate entropy and recurrence plots. The results of non-linear are displayed by pressing the nonlinear button on the top of the results view segment. The nonlinear results of HRV are shown in Figure 7.

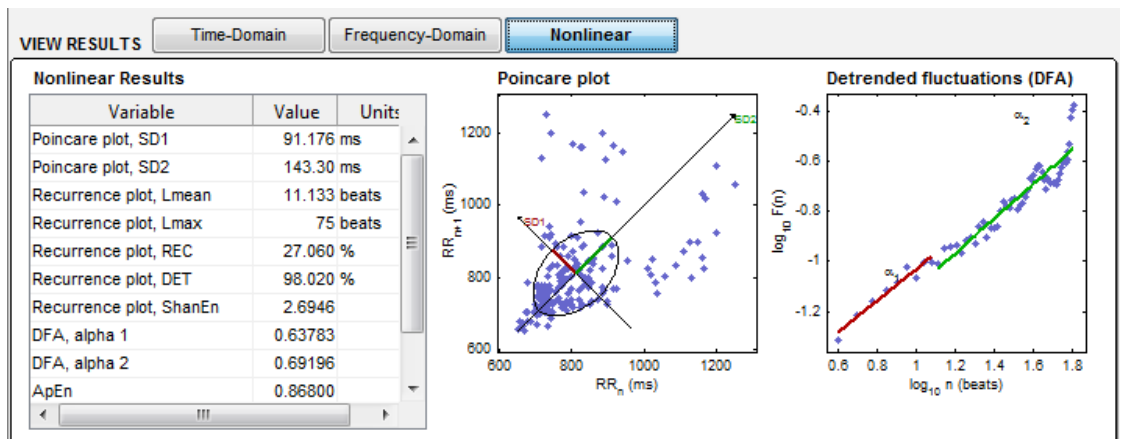

Figure 7: Results of nonlinear analysis of ECG signal.

Our investigation demonstrated that overall HRV is markedly depressed in alcoholic subjects. Alcoholics had lower values for time-domain and frequency domain parameters than controls (Table 1). 
Table1:Heart rate variability parameters in alcoholics group and in control group.

\begin{tabular}{|c|c|c|}
\hline Parameter & Control group & Alcoholics group \\
\hline $\begin{array}{c}\text { HR } \\
(\text { mean } \pm \text { SD })\end{array}$ & $67 \pm 5$ & $85 \pm 8$ \\
\hline $\begin{array}{c}\text { SDNN }(\mathrm{ms}) \\
(\text { mean } \pm \text { SD })\end{array}$ & $152 \pm 30$ & $112 \pm 35$ \\
\hline $\begin{array}{c}\text { RMSSD } \\
(\text { mean } \pm \text { SD })\end{array}$ & $122.4 \pm 35.0$ & $54.6 \pm 41.5$ \\
\hline $\begin{array}{c}\text { LF }(\mathrm{ms} 2) \\
(\text { mean } \pm \text { SD })\end{array}$ & $910 \pm 350$ & $516 \pm 254$ \\
\hline $\begin{array}{c}\text { HF }(\mathrm{ms} 2) \\
(\text { mean } \pm \text { SD })\end{array}$ & $490 \pm 113$ & $201 \pm 127$ \\
\hline $\begin{array}{c}\text { LF } / \mathrm{HF} \\
(\mathrm{mean} \pm \mathrm{SD})\end{array}$ & $6.8 \pm 4.2$ & $8.1 \pm 3.6$ \\
\hline
\end{tabular}

HRV parameters were significantly lower in the alcoholic's subjects. From Table II, we can see that the scaling exponent for normal subjects is around one and for alcoholics subjects, it is away from one. Power law correlation in signal fluctuation and opposite heart condition of the two types of subjects under study, normal and alcoholics subjects, is reflected clearly from the scaling exponent value. So, by Detrended Fluctuation technique, we can easily differentiate between normal and alcoholic personnel.

Table II: Detrended Fluctuation Analysis: Value of Scaling Exponent for 2 data sets (Normal and alcoholics subjects).

\begin{tabular}{|c|c|}
\hline $\begin{array}{c}\text { Normal Subjects } \\
\text { (Scaling exponent } \\
\boldsymbol{\alpha} \text { ) }\end{array}$ & $\begin{array}{c}\text { Alcoholics Subjects } \\
\text { (Scaling exponent } \boldsymbol{\alpha} \text { ) }\end{array}$ \\
\hline 0.9275 & 2.2087 \\
\hline 1.1232 & 2.4274 \\
\hline 0.8766 & 2.0395 \\
\hline 0.9024 & 1.9522 \\
\hline 0.9629 & 1.5944 \\
\hline 1.2895 & 1.7859 \\
\hline 0.9105 & 1.5989 \\
\hline 0.9630 & 2.0194 \\
\hline 1.1545 & 1.9293 \\
\hline
\end{tabular}


Table III: ApEn Analysis: Value of ApEn for 2 data sets (Normal and alcoholics subjects).

\begin{tabular}{|c|c|}
\hline $\begin{array}{c}\text { Normal Subjects } \\
\text { (Value of ApEn) }\end{array}$ & $\begin{array}{c}\text { Alcoholics Subjects } \\
\text { (Value of ApEn) }\end{array}$ \\
\hline 1.8517 & 0.8635 \\
\hline 1.6756 & 0.6054 \\
\hline 1.8413 & 0.7950 \\
\hline 1.7147 & 0.3412 \\
\hline 1.9521 & 0.8502 \\
\hline 1.7512 & 0.6782 \\
\hline 1.7815 & 0.7674 \\
\hline 1.8373 & 0.1237 \\
\hline 1.8932 & 0.0154 \\
\hline
\end{tabular}

From Table III, it is seen that normal subjects have higher ApEn value and alcoholics subjects have lower ApEn, thus clearly distinguishing the two groups. Disorder in the Heart rate signal and opposite heart condition of the two types of subjects under study, normal and alcoholics, is reflected clearly from ApEn value. So, by Approximate Entropy Method, we can easily differentiate between normal and alcoholics subjects.

Table IV: Poincare Plot Analysis for 2 data sets (Normal and alcoholics subjects).

\begin{tabular}{|c|c|c|c|}
\hline $\begin{array}{c}\text { Normal Subjects } \\
\text { (Value of SD1) }\end{array}$ & $\begin{array}{c}\text { Normal } \\
\text { Subjects } \\
\text { (Value of } \\
\text { SD2) }\end{array}$ & $\begin{array}{c}\text { Alcoholics Subjects } \\
\text { (Value of SD1) }\end{array}$ & $\begin{array}{c}\text { Alcoholics } \\
\text { Subjects } \\
\text { (Value of } \\
\text { SD1) }\end{array}$ \\
\hline 68 & 88 & 124 & 143 \\
\hline 57 & 91 & 119 & 138 \\
\hline 82 & 87 & 115 & 155 \\
\hline 73 & 84 & 130 & 147 \\
\hline 64 & 101 & 108 & 157 \\
\hline 55 & 92 & 117 & 163 \\
\hline 78 & 83 & 129 & 152 \\
\hline 75 & 98 & 132 & 139 \\
\hline 61 & 83 & 127 & 146 \\
\hline
\end{tabular}

From table IV it is seen that the values of SD1 and SD2 are lesser in normal subjects comparatively to the alcoholic subjects. The Poincare plot can also be visually analysed and the points under the ellipse shows the significant changes between the normal and alcoholic subjects.

\section{Conclusion and future perspectives}

These changes reflect significant reductions in cardiac parasympathetic activity and, possibly, increased sympathetic tone. As cardiovascular regulation mechanism is a nonlinear process, nonlinear methods, like Detrended Fluctuation Analysis, Approximate Entropy Method and Poincare Plot, may provide more powerful prognostic information than the traditional HR variability indexes. In this work, a comparative study is done on different techniques to distinguish HRV of normal subjects and alcoholics subjects. It has been demonstrated that both of 
these two nonlinear methods can successfully differentiate between two types of data and reflect the opposite heart condition of the two types of subjects under study, normal and alcoholics. Thus, value of the nonlinear parameters found in this work can be used as standard in detecting alcohol using HRV. Also, by measuring these nonlinear parameter values, a qualitative idea of heart condition can be obtained. HRV can forecast the illness independently and can also beyond that predict the risk factors for the traditional diseases. The work should be further carried on for all age groups and with various alcoholic related diseases.It will be of good scope and great interest to correlate the parameters of HRV with markers of increased sympathetic tone, like serum levels of catecholamines.

\section{REFERENCES}

[1] H. Kantz, J. Kurths, G. Mayer-Kress, Nonlinear Analysis of Physiological Data, 1st ed., Berlin, Germany: Springer, 1998.

[2] M. Kobayashi, T. Musha, "1/f fluctuation of heart beat period," IEEE Trans. Biomed. Eng. , vol. 29, pp. 456-457, Jun. 1982.

[3] S. M. Pincus, "Approximate a entropy as a measure of system complexity," Proc. Nat. Acad. Sci. USA, vol. 88, pp. 2297-2301, Mar. 1991.

[4] C. K. Peng, S. Havlin, J. M. Hausdorf, J. E. Mietus, H. E. Stanley, A. L. Goldberger, "Fractal Mechanisms and Heart Rate Dynamics: Long-range Correlations and their Breakdown with Disease," Journal of Electro cardiology, vol. 28, supp. 1, pp. 59-65, 1995.

[5] Task Force of The European Society of Cardiology and The North American Society of Pacing and Electrophysiology. Heart rate variability guidelines. Standards of a measurement, physiological interpretation, and clinical use Eur Heart J 1996; 17, 354 - 381.

[6] Moody GB, Mark RG, Goldberger AL, PhysioNet: A web-based resource for a the study of physiologic signals, IEEE, 2001; 20 (3) p. 70-75.

[7] Malpas SC, Neural influences on cardiovascular variability:possibilities and a pitfalls. Am J Physiol. 2002; 282, p. H6-H20.

[8] Serhat Balcioglu, MD, Ugur Arslan, MD, Sedat Türkoglu, MD, Murat Özdemir, MD, and Atiye Çengel, MD - Heart Rate Variability and Heart Rate Turbulence in Patients With Type 2 Diabetes Mellitus With Versus Without a Cardiac Autonomic Neuropathy - Am J Cardiol 2007;100:890-893

[9] Chua $\mathrm{KC}$ et al. Cardiac state diagnosis using higher order spectra of a heart rate variability. J Med Eng Technol. 2008; 32(2):145-55.

[10] S. Hans-Gorge, "Wavelets and signal processing; an application-based introduction,"Newyork: Springer, 2005.

[11] Andr'e E. Aubert, Bert Seps and Frank Beckers, "Heart Rate Variability in Athletes",Laboratory of Experimental Cardiology, School of Medicine, K.U. Leuven, Leuven, Belgium, Sports Med 2003; 33 (12): 889-919.

[12] M. Brennan, M. Palaniswami, and P. Kamen. Do existing measures of Poincare plot geometry reflect nonlinear features of heart rate variability. IEEE Trans BiomedEng,48(11):1342\{1347, November2001. 
Authors

D. Maheshkumar. Completed B.E in Instrumentation Technology \& M.Tech. in BioMedical Instrumentation from Sri Jayachamarajendra College of Engineering, Mysore. He has 16 years of teaching experience and he also pursuing Ph.D from Jain University, Bangalore. Currently he is working as Assistant Professor in the dept. of Electronics and Inst. Engineering, JSSATE, Bangalore. His areas of interest are Biomedical Signal processing, Electronic Instrumentation and Smart Sensors. He is the member of ISOI and ISTE. . He has one International \& two National Journals .

Dr. S C Prasannakumar. Completed B.E \& M. Tech. degree from Jayachamarajendra College of Engg. Mysore, and completed Ph.D degree from Avinash Lingam University, Tamilnadu, India. He is currently working as Professor in the department of Electronics and Inst. Engineering, RVCE, Bangalore. He has 17 years of teaching and industrial experience and he is the Member of ISTE. His areas of interests are Signal Processing (Acoustic Signal Processing) \& Bio-Medical Instrumentation. He is the BOS \& BOE of

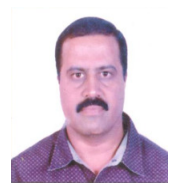
VTU and autonomous institutes. He has more than 28 publications in International \& National Journals and also in conferences.

Dr. B G Sudarshan. Completed MBBS degree from Sree Siddaratha Medical College, Tumkur and Ph.D in Bio-Medical Instrumentation from Avinash Lingam University, Tamilnadu. Currently he is working as Associate professor/medical officer at RVCE campus hospital/E\&IE and also Faculty in the Dept of Electronics and Inst. Engineering. He has more than 10 publications in International and National Journals \& also in conferences. He has more than 9 years of experience in this field and his area of interest are Bio-medical signal processing and Image processing.

Yadhuraj S.R. Completed B.E in Electronics and Communication \& M. Tech. degree from RVCE Bangalore, and pursuing PhD as a Research Scholar.
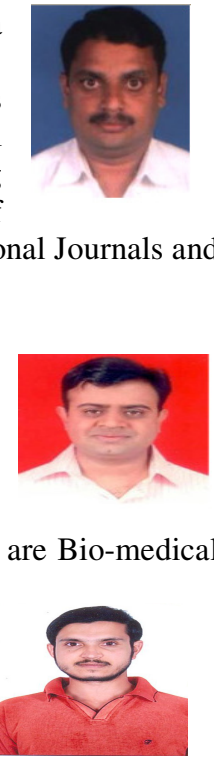